\title{
Mutations within the conserved NS1 nuclear export signal lead to inhibition of influenza A virus replication
}

\author{
Janne Tynell ${ }^{1 *}$, Krister Melén ${ }^{1}$ and Ilkka Julkunen ${ }^{1,2}$
}

\begin{abstract}
Background: The influenza A virus NS1 protein is a virulence factor and an antagonist of host cell innate immune responses. During virus infection NS1 protein has several functions both in the nucleus and in the cytoplasm and its intracellular localization is regulated by one or two nuclear localization signals (NLS) and a nuclear export signal (NES).
\end{abstract}

Methods: In order to investigate the role of NS1 NES in intracellular localization, virus life cycle and host interferon responses, we generated recombinant A/Udorn/72 viruses harboring point mutations in the NES sequence.

Results: NS1 NES was found to be inactivated by several of the mutations resulting in nuclear retention of NS1 at late stages of infection confirming that this sequence is a bona fide functional NES. Some of the mutant viruses showed reduced growth properties in cell culture, inability to antagonize host cell interferon production and increased p-IRF3 levels, but no clear correlation between these phenotypes and NS1 localization could be made. Impaired activation of Akt phosphorylation by the replication-deficient viruses indicates possible disruption of NS1-p85 $\beta$ interaction by mutations in the NES region.

Conclusion: We conclude that mutations within the NS1 NES result in impairment of several NS1 functions which extends further from the NES site being only involved in regulating the nuclear-cytoplasmic trafficking of NS1.

Keywords: NS1, NES, Influenza A virus

\section{Background}

Influenza A virus has a segmented genome consisting of eight single stranded negative-sense RNA molecules, which encode for 14 different proteins. Some of these proteins, such as the HA, PB1-F2 and NS1 proteins contribute to the virulence of the virus [1]. The $26 \mathrm{kDa}$ NS1 protein is a remarkably multifunctional protein. Through protein-RNA and protein-protein interactions NS1 is capable of preventing the production of interferons in virusinfected host cells and inhibiting the antiviral actions of interferon-induced proteins (reviewed by [2]). The inhibition of interferon production is facilitated by the ability of NS1 to inhibit the functions of a cytoplasmic RNA sensor RIG-I through interaction with TRIM25 [3]. In addition,

\footnotetext{
* Correspondence: janne.tynell@thl.fi

'Virology Unit, Department of Infectious Disease Surveillance and Control, National Institute for Health and Welfare (THL), Mannerheimintie 166, FIN-00300 Helsinki, Finland

Full list of author information is available at the end of the article
}

the C-terminal effector domain of NS1 can bind and inhibit the nuclear proteins CPSF30 and PABII, which function in the 3 '-end processing of cellular pre-mRNAs $[4,5]$. The inhibition of interferon-induced dsRNA-activated proteins PKR and 2' -5 '-oligoadenylate synthetase (OAS) is mediated through direct binding of NS1 to PKR [6] and through sequestering of dsRNA from OAS by the $\mathrm{N}$ terminal RNA-binding domain of NS1 [7]. Moreover, NS1 has been suggested to inhibit the host RNAi pathway [8] and it is also involved in many regulatory functions such as controlling the host cell splicing machinery $[9,10]$, enhancing the translation of viral mRNA [11] and activating the PI3K pathway [12].

Since NS1 has many functions both in the nucleus and in the cytoplasm, it is clear that the intracellular localization of NS1 must be controlled accordingly. Structural and functional studies of the NS1 protein have recognized an $\mathrm{N}$-terminal nuclear localization signal (NLS1) involving 
amino acids Arg-35, Arg-38 and Lys-41 [13], a nuclear export signal (NES) within amino acids 138-147 [14] and a C-terminal nuclear/nucleolar localization signal (NLS2/ NoLS) comprising of the basic amino acids at positions 219, 220, 224 and 229 and including also amino acids 231 and 232 in NS1 proteins which have a C-terminal extension $[15,16]$. Localization studies have shown that NS1 localizes predominantly in the nucleus, but it is translocated into the cytoplasm at late stages of infection possibly due to the unmasking of NES or, in the event of a competitive relationship between NLS and NES, perhaps because of masking of the NLS signals.

All NS1 proteins have a putative well-conserved NES, which is characterized as a classical hydrophobic leucinerich export signal consisting of the consensus sequence $\Phi$ $\mathrm{XX}(\mathrm{X})-\Phi-\mathrm{XX}(\mathrm{X})-\Phi-\mathrm{X}-\Phi$, where $\Phi$ represents L, I, F, V or $M$ and $X$ represents any amino acid. Leucine-rich NESs are typically recognised by the chromosome region maintenance 1 (CRM1) exportin molecule, which directly binds to NES and mediates the transport of NES-containing molecules through the nuclear pore complex in a NES-CRM1Ran-GTP ternary complex (reviewed by [17]). Recently it was suggested that some leucine-rich NES-containing proteins may be translocated into the cytoplasm independently of CRM1 $[18,19]$. Accordingly, a FRET analysis of transiently expressed NS1 protein failed to demonstrate a direct interaction of NS1 with CRM1 [20]. However, since the NES of ectopically expressed NS1 has been reported to remain inactive in uninfected cells [14], this observation has to be verified in the context of live virus infection.
In the present study we generated recombinant influenza A viruses with mutations in the putative NES of NS1 protein. We show that mutations in the NES of NS1 molecule results in nuclear retention of the NS1 protein at late stages of infection. In addition, we obtained mutant viruses that had a reduced capacity to replicate in cell culture and showed enhanced activation of IRF3 and production of antiviral interferons (IFN). Lack of efficient Akt phosphorylation by the growthdeficient viruses suggests impairment of the NS1-p85 $\beta$ interaction by mutations within the NES.

\section{Results}

The NS1 nuclear export signal is well-conserved within the majority of influenza A virus strains

A schematic presentation of the NS1 protein structure (A/Udorn/72 virus NS1) is shown in Figure 1. The NS1 NES is located in the middle of the effector domain and comprises of a phenylalanine and three leucines each separated by one or two amino acids (Figure 1A). Within the dimeric structure of the effector domain the NES sequences are partially buried in the molecule (Figure 1C). In order to determine the conservation rate of the NS1 NES we compared more than 25,000 influenza A NS1 amino acid sequences available (as of September 2013) in the Influenza Research Database [21] using the sequence variation analysis tool accessible on the website http://www.fludb.org (Table 1). The NES consensus sequence (F138, L141, L144 and L146) appears to be highly conserved within all influenza A strains regardless of host

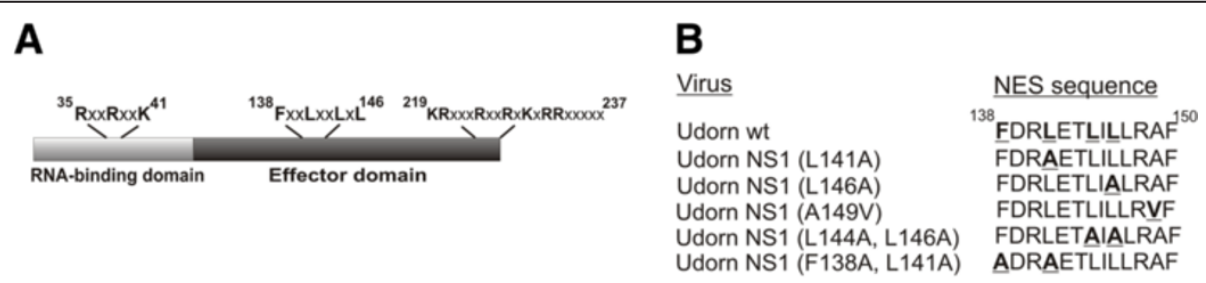

C

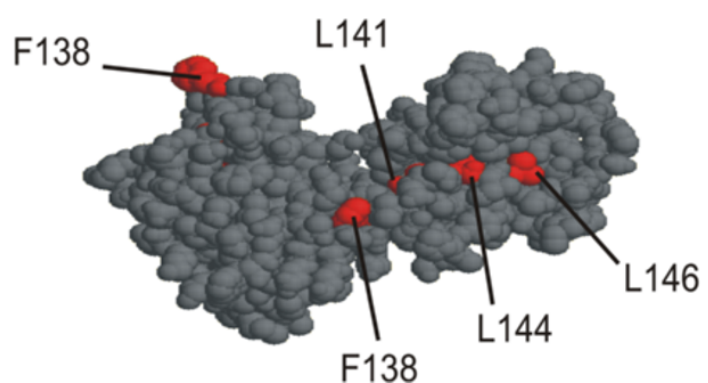

Figure 1 The nuclear export signal of A/Udorn/72 NS1. A, schematic representation of A/Udorn/72 NS1 with the localization signals NLS1 (aa 35-41), NES (aa 138-146) and NLS2/NoLS (aa 219-232) indicated. B, sequence of A/Udorn/72 NS1 NES region and the mutations introduced into the NES sequence to create recombinant mutant viruses. C, molecular surface representation of the position of NES within the dimeric A/Udorn/72 NS1 effector domain based on the crystal structure by Xia et al. [22] (PDB ID 3EE8). Amino acids of the NES consensus sequence are indicated in red. The figure was created using RasMol 2.7.3. program (http://rasmol.org/). 
Table 1 Amino acid conservation of the NS1 NES region

\begin{tabular}{llll}
\hline Position & Consensus & Prevalence & Percentage \\
\hline 138 & Phe & $24424 / 25183$ & $96,99 \%$ \\
139 & Asp & $15412 / 25183$ & $61,20 \%$ \\
140 & Arg & $22525 / 25183$ & $89,44 \%$ \\
141 & Leu & $24763 / 25183$ & $98,33 \%$ \\
142 & Glu & $24916 / 25183$ & $98,94 \%$ \\
143 & Thr & $22149 / 25183$ & $87,95 \%$ \\
144 & Leu & $21822 / 25183$ & $86,65 \%$ \\
145 & lle & $16910 / 25183$ & $67,15 \%$ \\
146 & Leu & $22655 / 25183$ & $89,96 \%$ \\
147 & Leu & $25105 / 25183$ & $99,69 \%$ \\
148 & Arg & $25099 / 25183$ & $99,67 \%$ \\
149 & Ala & $25134 / 25183$ & $99,81 \%$ \\
150 & Phe & $25092 / 25183$ & $99,64 \%$ \\
\hline A consen & Pen &
\end{tabular}

A consensus sequence for the region was derived by running 25183 NS1 sequences through the Analyze Sequence Variation (SNP) tool on the Influenza Research Database -website (http://www.fludb.org). Prevalence of each amino acid is indicated as a total number and percentage within all the analyzed sequences.

species. In addition, the region immediately adjacent to the export signal (aa 147-150) is also very highly conserved.

\section{Mutations in the putative nuclear export signal lead to nuclear retention of NS1 at late stages of infection}

In order to determine the key amino acids regulating the nuclear export of NS1 protein we created different A/ Udorn/72 mutant viruses with one or two point mutations introduced into the putative NES sequence (Figure 1B). In addition, we also generated NS1 A149V mutant virus, since this mutation has been linked to an attenuated phenotype in an avian influenza A virus [23]. The impact of the putative NES mutations on NS1 intracellular localization at different stages of infection was studied in A549 cells by immunofluorescence assay (Figure 2) followed by quantitative analysis of the intracellular localization of wild type and mutant NS1 proteins (Figure 3). At early stages of infection there were no marked differences between the localization patterns of the mutated proteins and the wildtype NS1 protein, since in all cases the protein was found both in the nucleus and the cytoplasm. At later stages of infection (16 $\mathrm{h}$ and later), however, most of the mutant NS1 proteins (L141A), (A149V), (F138A, L141A) and (L144A, L146A) accumulated in the nucleus leaving the cytoplasm almost or completely devoid of NS1 protein, while a significant proportion of the wild-type NS1 protein localized into the cytoplasm in addition to the nucleus. The NS1 L146A mutant protein behaved very similarly to that of the wt NS1 protein and thus this point mutation appears to be insufficient to fully inactivate the NES (Figures 2 and 3).

\section{Inactivation of the NS1 NES has limited impact on replication of the virus}

Next we analyzed the effect of NES mutations on the viral growth properties of different recombinant viruses. We infected MDCK cells with the wt and NES-mutant viruses at very low MOI values (0.001) and measured the virus titers from samples taken at $12 \mathrm{~h}$ intervals. The growth curves derived from the results showed no major differences in mutant virus titers as compared to the wild type virus, with the exception of the (A149V) and (L144A, L146A) NS1 mutant viruses (Figure 4). The growth of (L144A, L146A) NS1 mutant virus was strongly inhibited, presenting approximately 1000 -fold lower titers throughout the infection than the wt virus. Also the growth kinetics of (L144A, L146A) NS1 mutant virus was delayed with peak titer achieved only at $36 \mathrm{~h}$ after infection as compared to $24 \mathrm{~h}$ with all the other viruses. The (A149V) NS1 mutant virus displayed equal titers with wt virus at the $12 \mathrm{~h}$ time point, but afterwards only grew to $10-100$ fold lower titers than the wt virus. The observed reduction in virus production is not directly linked with the inactivation of the NES, since (L141A) and (F138A, L141A) NS1 mutant viruses show clear inactivation of NES and accumulation of NS1 protein in the nucleus (Figure 2) and yet they replicated very well in MDCK cells. To investigate whether the observed growth defects correlated with any deficiencies in viral protein expression, we infected A549 cells at MOI 1 and analyzed the expression of NP, NS1, $\mathrm{M} 1$ and NEP in cell lysate samples collected at $2 \mathrm{~h}, 4 \mathrm{~h}$, $6 \mathrm{~h}, 8 \mathrm{~h}, 12 \mathrm{~h}$ and $24 \mathrm{~h}$ post-infection by Western blotting (Figure 5). All viruses displayed largely similar viral protein expression kinetics throughout the infection (Figure 5), but there was a clear reduction in the levels of NS1 and NEP production by the (L144A, L146A) mutant virus and some less pronounced variation among the other viruses. NEP mediates viral ribonucleoprotein (vRNP) export [24], so we checked for any differences in vRNP localization by visualizing NP localization by immunofluorescence in infected A549 cells (Additional file 1: Figure S1 and Additional file 2: Figure S2), but found no differences between the viruses.

\section{The NS1 mutations A149V and L144A, L146A lead to increased interferon production}

In order to analyse whether the reduced replication of (A149V) and (L144A, L146A) mutant viruses is the result of an impaired ability of NS1 protein to block host cell interferon production, we infected A549 cells with the 5 mutant viruses and the wild-type virus at different MOI values and measured the production of IFN- $\beta$ and IFN- $\lambda 1$ into the supernatants at $20 \mathrm{~h}$ post infection (Figure 6A and $\mathrm{B})$. Recombinant wild type $\mathrm{A} / \mathrm{Udorn} / 72$ virus and NS1 (L141A), (L146A) and (F138A, L141A) mutant viruses were very weak in their ability to induce IFN- $\beta$ and 


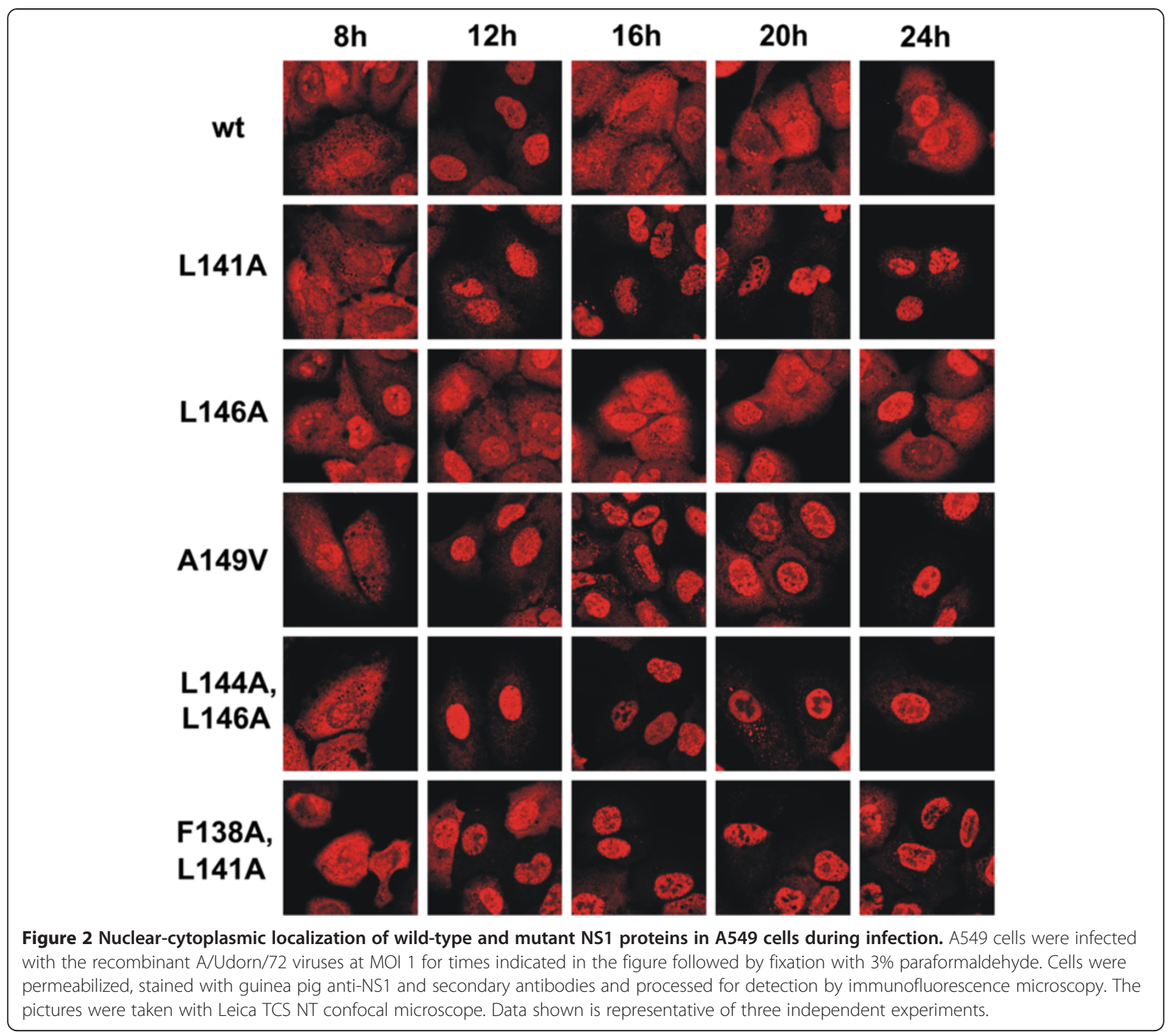

IFN- $\lambda 1$ production into the cell culture supernatant. However, NS1 (A149V) and especially NS1 (L144A, L146A) mutant viruses readily induced relatively high IFN levels in response to the infection (Figure 6A and $\mathrm{B}$ ). Consistent with the ELISA data, Western blot analysis of cell lysates from virus-infected cells showed high levels of phosphorylated form of IRF3 (p-IRF3) and enhanced expression of MxA protein for the NS1 (A149V) and (L144A, L146A) viruses (Figure 6C). Interestingly, NS1 (L141A), (L146A) and (F138A, L141A) mutant viruses were also able to induce stronger IRF3 phosphorylation than the wt virus and detectable levels of MxA (Figure 6C), even though only low levels of IFN were produced by these viruses (Figure 6A and B). Since the phosphaditylinositol-3-kinase (PI3K) pathway is also activated and regulated by NS1 protein during influenza virus infection [12], we analyzed whether NES mutations would also have an effect on Akt phosphorylation. Interestingly, the amount of p-Akt within the infected cells was clearly reduced in the NS1 (A149V) and (L144A, L146A) mutant virus infected cells, suggesting an impairment of the PI3K activating functions of NS1. A notable feature was also the clear reduction in NS1 levels for the mutant virus (L144A, L146A) (Figure 6C), indicating a possible impairment of protein stability caused by the mutations. For better comparison of NS1 expression levels, a new Western blot analysis with further diluted samples was performed and band intensities were analyzed with ImageJ (http://imagej.nih.gov/ij/) software, revealing an almost 50\% reduction in NS1 (L144A, L146A) expression levels as compared to wild type virus (Additional file 3: Figure S3). 


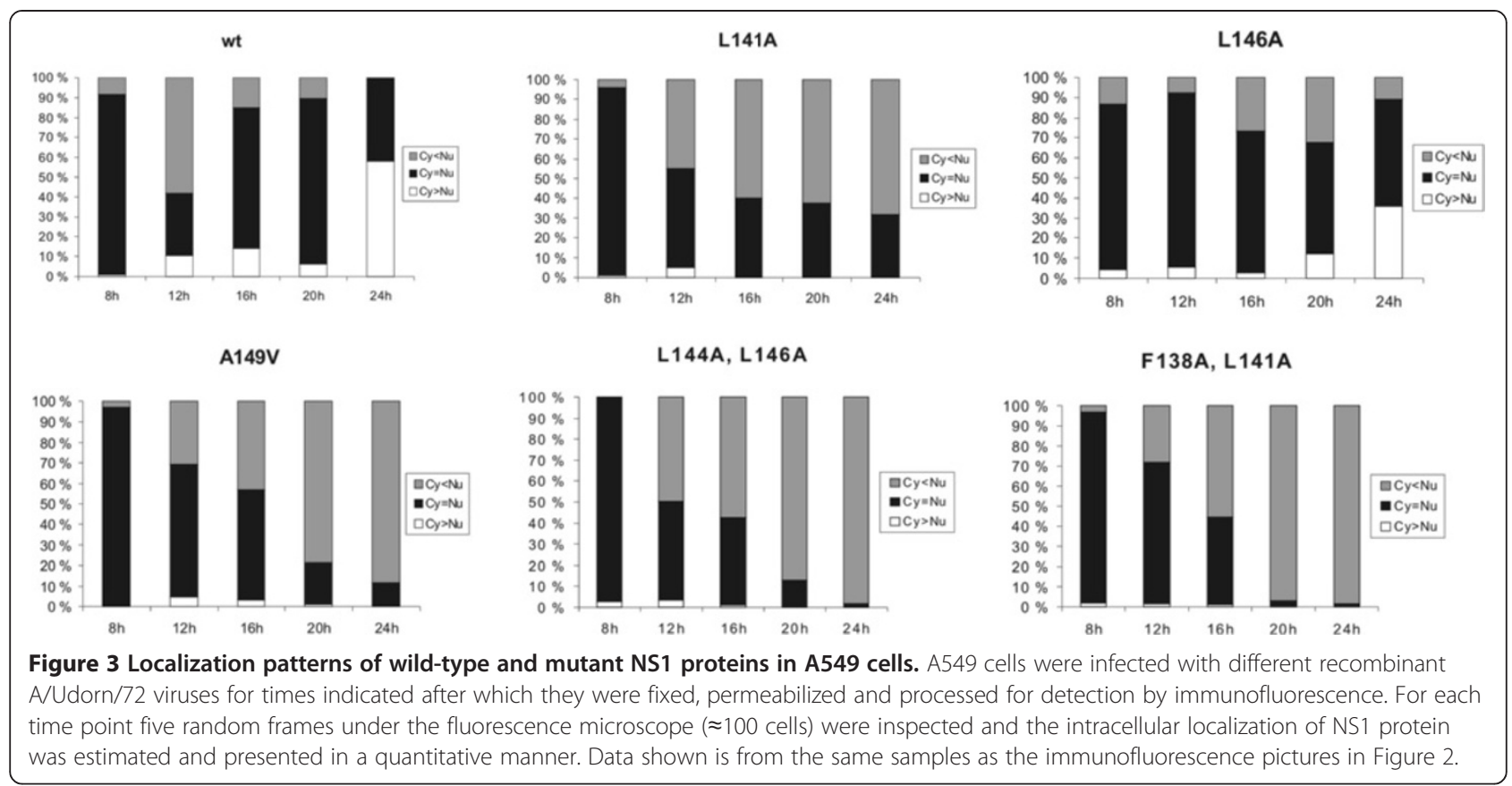

Interferon production by A149V and L144A, L146A mutant viruses is not due to inability of NS1 to bind CPSF The NES-region of NS1 has also been proposed to play a role in CPSF binding [25]. In order to study whether the observed increase in interferon production was due to an inability of the NS1 protein to bind CPSF, we created the mutations (A149V), (L144A, L146A) and (F138A, L141A) into GST-NS1 expression constructs and compared their ability to bind CPSF30 to that of wild-type NS1 in a GST pull-down assay combined with autoradiography (Figure 7). As shown in Figure 7, all the mutant GST-NS1 proteins

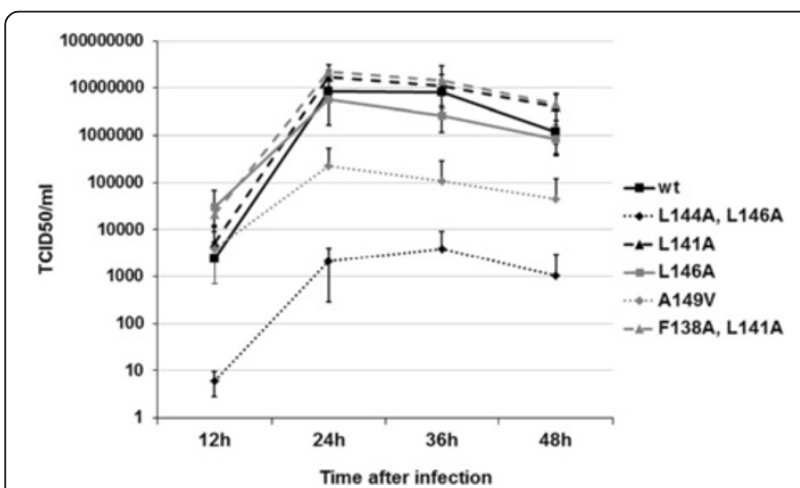

Figure 4 Growth kinetics of the recombinant viruses in MDCK cells. MDCK cells on 6-well plates were infected at MOI values of 0.001 for $1 \mathrm{~h}$ after which virus inoculum was removed and fresh growth medium was added. The cells were incubated at $37^{\circ} \mathrm{C}$ humidified $5 \% \mathrm{CO}_{2}$ conditions and supernatant samples were taken at $12 \mathrm{~h}$ intervals and analyzed by endpoint dilution on MDCK cells. Mean values from three independent experiments are shown for each sample. bind CPSF30 equally well and thus we can conclude that the NES mutations are unlikely to have an effect on NS1 CPSF-binding.

\section{NS1 protein export during influenza $A$ infection is likely} not mediated by CRM1

The nuclear export of NES-containing proteins is often regulated by the nuclear export protein, CRM1. An antifungal antibiotic, leptomycin B (LMB) is able to directly bind to CRM1 [26] and thus block NES-dependent nuclear export of proteins. In order to analyze whether the nuclear export of influenza A virus NS1 protein is dependent on CRM1 we treated wt virus-infected cells with LMB and analyzed the intracellular localization of NS1 protein by immunofluorescence at $20 \mathrm{~h}$ post infection (Figure 8). As a positive control we included the analysis of viral NP, since its export from the nucleus has previously been shown to be dependent on CRM1 [27]. For a quantitative assessment 6-8 random frames $(\approx 100$ cells) were inspected under the microscope and NS1 and NP localization was estimated and presented in a graph (Figure 8). NP was clearly found to be retained in the nucleus in LMB-treated cells confirming the successful inhibition of CRM1 functions. NS1, however, showed little difference in localization between LMBand mock-treated cells with most cells displaying NS1 both in the nucleus and the cytoplasm. This suggests that LMB treatment was inefficient in inhibiting the nuclear export of NS1 indicating that the export of this protein is likely independent or only partially dependent on CRM1. 


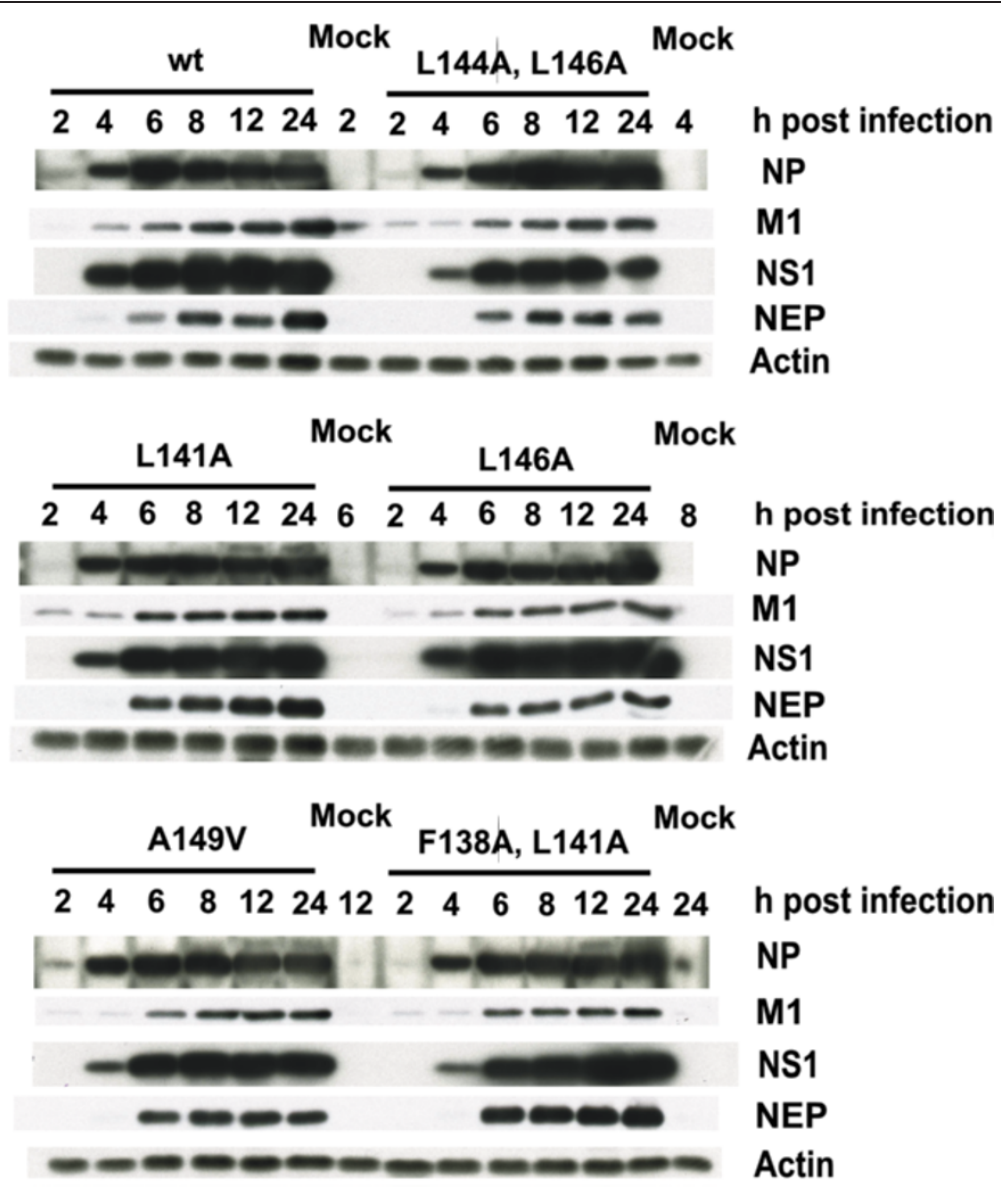

Figure 5 Expression kinetics of viral proteins during infection. A549 cells were infected at MOI 1 with A/Udorn/72 wild type and mutant viruses and cell lysates were collected at the indicated time points. The amounts of NS1, NP, NEP, M1 and actin in the lysates were visualized by immunoblotting (10 $\mathrm{\mu g}$ total protein/lane). Data shown is representative of three independent experiments.

\section{Discussion}

In the present study we have carried out a detailed functional analysis of influenza A virus NS1 protein NES element, which is extremely well-conserved among all presently known influenza A virus strains and represents a typical consensus NES-type sequence. We show that mutations in the NES element, with the exception of mutation L146A, lead to nuclear retention of NS1 at late stages of infection, indicating an inactivation of the NES. NES-inactivating NS1 mutations (L144A, L146A) and (A149V) were associated with severe inhibition of viral growth properties and inability of the mutant virus to inhibit host cell interferon production.

Regulation of the mechanisms of NS1 NES function during infection is unknown, although the amino acid region immediately after the consensus-like NES element has been suggested to play a regulatory role in the nuclear export of NS1 [14]. This may take place by masking of the export signal at early times of infection and by allowing the exposure of the NES element at later time points by some activating event. The fact that some activation event is required for triggering the nuclear export of NS1 protein is well supported by the structure of NS1 (Figure 1C). Leucine residues in the NES element are of hydrophobic nature and for them to become in contact with export molecules relatively drastic conformation changes are required. Inactivation of the NES by the A149V mutation, which renders the protein strongly nuclear at late time points (Figure 2), also supports the view that some masking sequence is regulating the accessibility of the consensus leucine residues to the export machinery. Interestingly, the A149V NS1 mutation has been linked to pathogenesis in chickens when it was discovered in the context of a natural $\mathrm{H} 5 \mathrm{~N} 1$ virus strain that was circulating in poultry [23].

The observed reduction in viral replication of the NS1 mutant viruses (A149V) and (L144A, L146A) (Figure 4) was likely due to impaired IFN antagonist functions of mutant NS1 proteins. These viruses readily induced IFN production into cell culture supernatant, as evidenced by the ELISA assays (Figure 6A and B), and also showed induction of IFN induced MxA protein (Figure 6C). Upon 


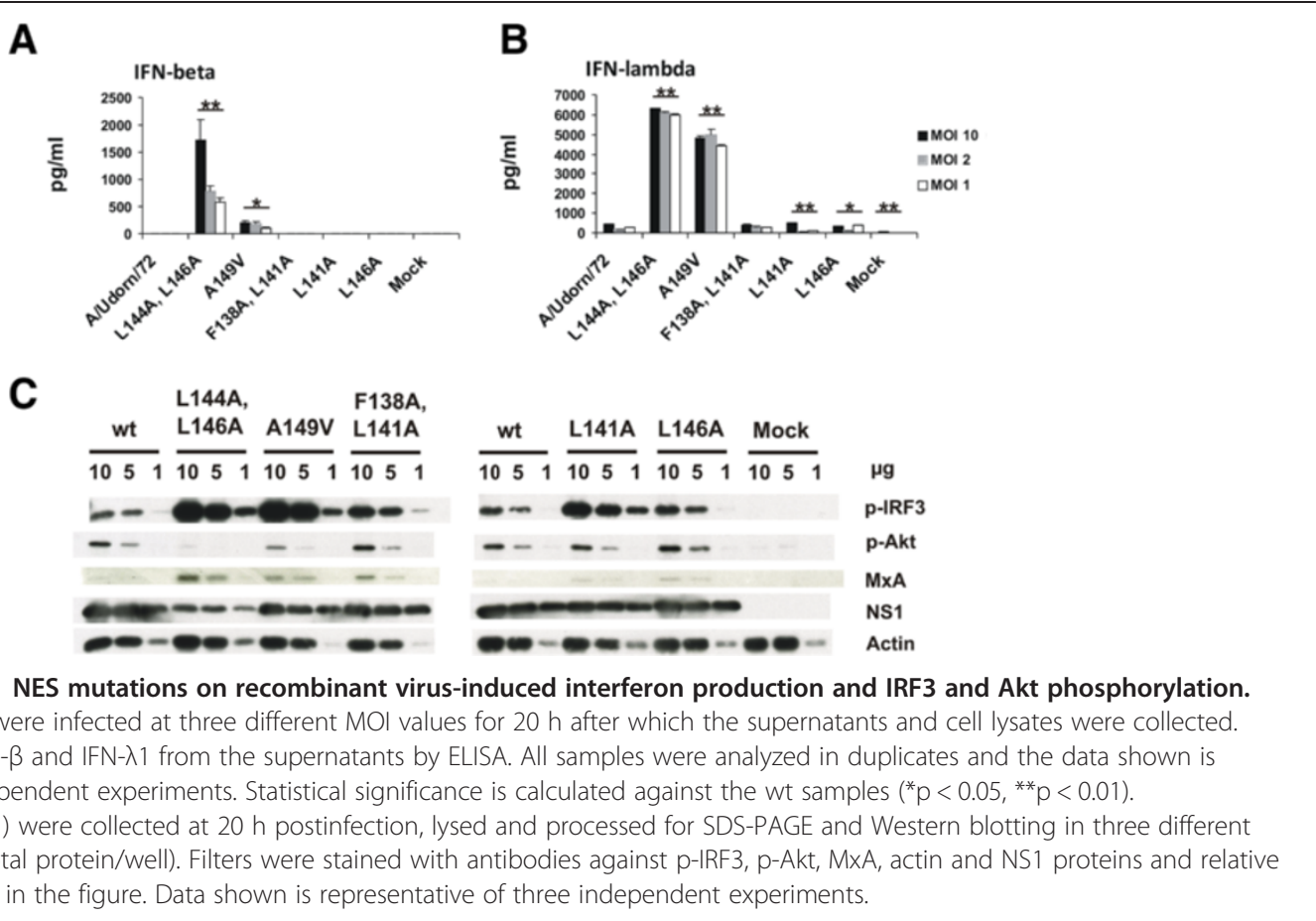

further investigation the elevated IFN levels were found to correlate with very high virus activated p-IRF3 levels as compared to wild-type virus, suggesting the inability of these mutant NS1 proteins to inhibit the RIG-I pathway. Strong expression of p-IRF3 was also observed with the other nuclearly localized NS1 mutant viruses (L141A) and (F138A, L141A), which might be explained by the nuclear NS1 being unable to inhibit cytoplasmic RIG-I. However, this correlation remains unclear since strong phosphorylation of IRF-3 was also associated with the L146A NS1 mutant virus, which shows a similar NS1 localization pattern as the wild type virus. Lack of potent IFN production despite strong p-IRF3 activation by the (L141A) and (F138A, L141A) NS1 mutant viruses indicates, that in the case of at least some nuclearly retented mutant NS1 proteins, the nuclear functions of NS1 in the absence of cytoplasmic NS1 are sufficient to inhibit IFN production. Detectable levels of MxA were observed with all the mutant viruses despite low IFN production by some of them, which is probably due to even low IFN production being enough to cause some MxA production. It was also of interest that Western blot analysis revealed diminished

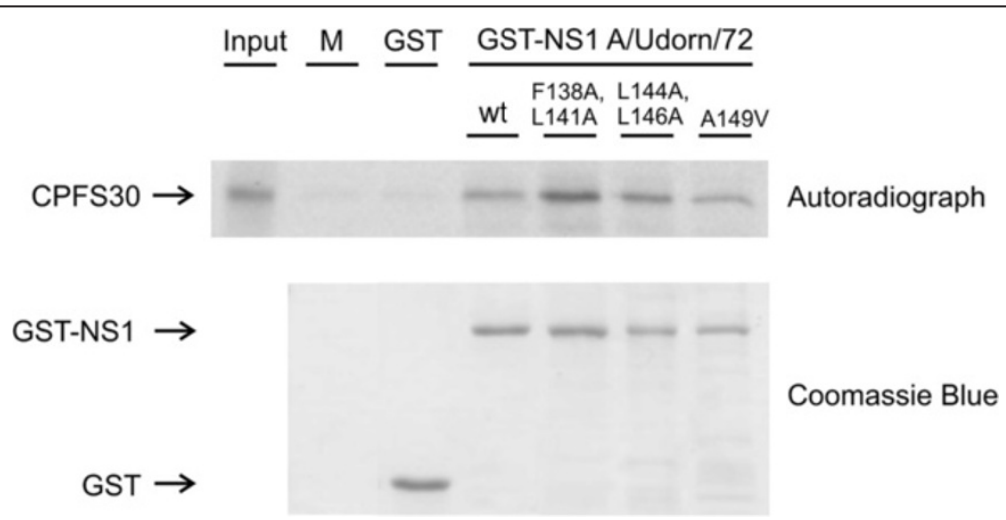

Figure 7 The nuclear export -deficient mutants of the full length influenza A/Udorn/72 NS1 protein bind in vitro-translated CPFS30 protein in a GST pull down experiment. ${ }^{35} \mathrm{~S}$ ]-labeled and in vitro-translated CPFS30 was allowed to bind to E. coli-expressed and Sepharoseimmobilized GST, GST-NS1 A/Udorn/72 wt, GST-NS1 F138A, L141A, GST-NS1 L144A, L146A and GST-NS1 A149V at + $4^{\circ} \mathrm{C}$ for $1 \mathrm{~h}$. Sepharose-bound proteins were dissolved in Laemmli sample buffer, separated on 12\% SDS-PAGE and autoradiographed. Input, lane C, was 1:20 of the amount of $\left.{ }^{35} \mathrm{~S}\right]$-labeled protein that was used in binding experiment. M indicates the Sepharose matrix-bound proteins. The same samples were also separated on 12\% SDS-PAGE and stained with Coomassie Blue to visualize the amount of Sepharose-immobilized GST and GST-NS1 fusion proteins. 


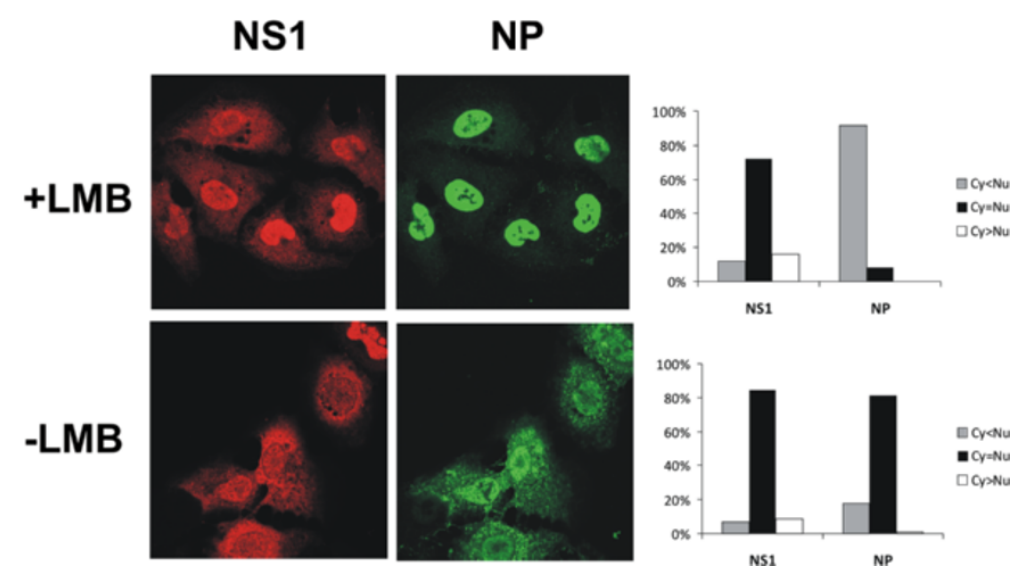

Figure 8 Effect of leptomycin B treatment on NS1 intracellular localization. A549 cells were infected with wild-type A/Udorn/72 virus and incubated with or without $2 \mathrm{ng} / \mathrm{ml} \mathrm{LMB}$ added at $3 \mathrm{~h}$ postinfection. $20 \mathrm{~h}$ postinfection the cells were fixed and the intracellular localization of NS1 protein and NP were visualized by immunofluorescence and estimated in a quantitative manner by observing 6-8 random frames under the microscope. The pictures were taken by Leica TCS NT confocal microscope. Data shown is representative of three independent experiments.

p-Akt levels for the (L144A, L146A) and (A149V) mutants adding further evidence that multiple NS1 functions may be affected by the mutations. This suggests a more extensive structural or functional importance of the NES region. In a study investigating the structural conservation of the whole NS1 protein, Leu144 and Ala149 were found to be very well-conserved and were suggested to play a role in stabilizing the protein structure [28]. The observed reduction of almost $50 \%$ in the levels of NS1 (L144A, L146A) compared to wild type NS1 (Additional file 3: Figure S3) supports the idea of a stabilizing role for the NES region, but this is unlikely to have an impact on viral growth properties or IFN production since very low levels of NS1 have been shown to be sufficient for normal replication and IFN antagonism [29]. The NES region has also been suggested to be involved in CPSF binding [25], but an X-ray crystal structure published on the NS1-CPSF complex somewhat later does not support this view [30]. Nevertheless, we analyzed whether any of our inserted mutations affected CPSF-binding of NS1 on GST pull-down assay and found that binding to CPSF30 was similar to that of wild-type NS1 (Figure 7).

Besides being involved in regulating splicing and nuclear export of mRNA [10], NS1 has also been shown to influence temporal regulation of viral RNA and protein synthesis [31]. Thus we explored whether the introduced NES mutations might have an effect on viral protein expression kinetics (Figure 5). In addition to NS1, we included an abundant early gene product (NP), a late gene product (M1) and a spliced gene product (NEP). At a glance, expression kinetics of all the analyzed proteins appear similar between the wild type and mutant viruses (Figure 5), but there are some differences in expression levels at certain time points. The growth deficient viruses (A149V) and especially (L144A, L146A) produce somewhat lower levels of NS1 and NEP, whereas the (L141A) and (L146A) viruses are even stronger inducers of NEP than the wild type virus (Figure 5). NP and M1 expression levels are mostly equal between the different viruses with the exception of (A149V), (L146A) and (F138A, L141A) viruses showing slightly reduced M1 levels. All the observed differences are modest however, and despite critical roles for these proteins during infection, unlikely to cause any defects in replication. To confirm this conclusion we used immunofluorescence analysis to visualize NP localization during infection with all our mutant viruses (Additional file 1: Figure S1 and Additional file 2: Figure S2). Since NEP and M1 are involved in the nuclear export of viral ribonucleoproteins $[24,32]$, any major flaw in their function would show as nuclear retention of NP. All the viruses display equal cytoplasmic localization of NP (Additional file 1: Figure S1 and Additional file 2: Figure S2), so we can conclude that the observed growth deficiencies are not due to any defects in viral mRNA splicing or protein expression.

The PI3K pathway has a dual role in influenza A virus infection by mediating processes that are both beneficial and detrimental for virus replication. Several studies have shown that the PI3K pathway supports viral replication by promoting viral entry [33], inhibiting apoptosis [34-36] and enhancing nuclear export of vRNP complexes [37]. In addition, PI3K signalling is also involved in RIG-I mediated activation of IRF3 thus contributing to the induction of type I interferon production [38]. PI3K is activated at two points during influenza A infection, first at a very early stage stimulated by viral attachment and then at a later stage of infection by NS1 binding to the $\mathrm{p} 85 \beta$ subunit of PI3K [33]. NS1 has also been suggested to directly bind to Akt, a downstream 
effector of PI3K involved in diverse cellular processes including cell differentiation, proliferation and apoptosis [39]. Diminished levels of cellular p-Akt observed in (L144A, L146A) and (A149V) mutant virus-infected cells point towards defects in the NS1 PI3K activating functions (Figure 6C), which correlates with a previous observation of the region around NES being involved in PI3K activation [40]. Li and coworkers found the amino acid region 137142 to be involved in NS1-p85 $\beta$ interaction and showed interference of the interaction by mutation of the amino acids 141 and 142. In a structural study of the p $85 \beta$ iSH2NS1 -complex the Glu 142 of NS1 was speculated to be involved in salt bridge formation at the complex interface [41]. The inability of NS1 (L144A, L146A) and (A149V) mutant viruses to activate PI3K signalling could result from NS1 nuclear retention, but since this phenotype is not shared by other viruses with inactivated NS1 NES signals, it must be due to an effect independent of NS1 localization. It is plausible that mutations close to the NS1-p85 $\beta$ binding site could cause structural changes big enough to disrupt the salt bridge formation between NS1 Glu 142 and $\mathrm{p} 85 \beta$ or just influence the interaction enough to disrupt PI3K/Akt activation. Such disruption by itself could cause the observed reductions in growth and IFN antagonistic properties as evidenced by other studies where NS1-p85 $\beta$ interaction has been investigated [12,41,42]. Also certain mutations in the amino acid position 138 have been shown to influence the NS1-p85 $\beta$ interaction $[43,44]$, so it is feasible that the mutations L144A, L146A and A149V might affect it as well.

Nuclear export of proteins containing the classical leucine-rich export signal is usually mediated by CRM1, but with influenza A virus NS1 this is likely not the case. It was shown previously by transfection experiments that NS1 does not interact with CRM1 [20] and here we show that the CRM1 inhibitor LMB fails to prevent or at least partially impair NS1 export during the infection (Figure 8). Examples of proteins showing nuclear export independent of CRM1, despite harboring leucine-rich export signals, include protein kinase inhibitor (PKI) and glucocorticoid receptor, which are exported by the $\mathrm{Ca}^{2+}$-binding protein calreticulin through a mechanism similar to CRM1mediated export $[18,19]$. It is likely that a similar alternative export pathway exists for NS1 as well, but this remains to be verified by further experiments.

\section{Conclusions}

In conclusion, our data shows that the NES region of NS1 plays an extremely important role during influenza A virus infection. It was clearly shown that the NES sequence regulates the nucleo-cytoplasmic transport of NS1. An association with nuclear retention of NS1 and increased phosphorylation of IRF3 was observed, but no clear correlation could be established. There were obvious differences in the growth properties of the different NES mutant viruses, and since the observed NS1 localization pattern was a poor prognosis for the growth phenotype, it must be concluded that the importance of NS1 NES region goes beyond just regulating NS1 localization. Good correlation of Akt phosphorylation with growth properties indicates the involvement of NS1-p85 $\beta$ interaction, but conclusive identification of the molecular basis of these differences requires further studies.

\section{Methods}

\section{Cells and viruses}

HEK293, A549 and MDCK cells were maintained in Eagle's minimum essential medium (MEM) supplemented with $0.6 \mu \mathrm{g} / \mathrm{ml}$ penicillin, $60 \mu \mathrm{g} / \mathrm{ml}$ streptomycin, $2 \mathrm{mM} \mathrm{L-glu-}$ tamine, $20 \mathrm{mM}$ HEPES and 10\% fetal calf serum (FCS) at $37^{\circ} \mathrm{C}$ in $5 \% \mathrm{CO}_{2}$. Influenza A/Udorn/72 recombinant wildtype virus (wt) and recombinant NS1 NES -mutant viruses were propagated in 11-day-old embryonated chicken eggs at $34^{\circ} \mathrm{C}$ for 3 days.

\section{Generation of recombinant viruses}

The wild-type A/Udorn/72 virus and the recombinant NS1 NES-mutant viruses were generated using plasmidbased reverse genetics essentially as previously described [45]. pHH21 plasmids containing the full-length cDNAs for the eight A/Udorn/72 genomic RNA segments and pcDNA plasmids coding for the A/Udorn/72 proteins PB1, PB2, PA and NP were kindly provided by Dr. Robert A. Lamb. NES-mutations (L141A), (L146A), (A149V), (F138A, L141A) and (L144A, L146A) were introduced into the pHH21-NS plasmid using specific oligonucleotide primers and a QuikChange II Site-Directed Mutagenesis Kit (Stratagene, La Jolla, CA). ORF NS2 was not affected by any of the mutations. For the generation of recombinant viruses a co-culture of HEK293 and MDCK cells was co-transfected with $1 \mu \mathrm{g}$ of each of the 12 plasmids coding for the viral RNA segments and expression contructs using TransIT-LT1 transfection reagent (Mirus, Madison, WI). The transfected cells were maintained in serum-free MEM and at $24 \mathrm{~h}$ post-transfection 2,5 $\mathrm{\mu g} / \mathrm{ml}$ TPCKtrypsin was added into the culture medium. At 3 days post-transfection culture supernatants were harvested and viruses were propagated through two passages followed by plaque purification on MDCK cells. Individual plaques were then amplified in 11-day-old embryonated chicken eggs. NS genes of the recombinant viruses were sequenced to verify that the generated mutations were as expected.

\section{Antibodies}

Guinea pig anti-NS1 [15] and rabbit anti-NP antibodies [46] have been previously described. Rhodamine Red Xlabeled anti-guinea pig immunoglobulins and FITC-labeled anti-rabbit immunoglobulins were used as secondary 
antibodies in immunofluorescence analysis (1:100, Jackson ImmunoResearch Laboratories, Inc., West Grove, PA, USA). In Western blotting guinea pig anti-NS1 [15], rabbit anti-Actin (sc-10731; 1:500 dilution; Santa Cruz Biotechnology, Santa Cruz, CA), rabbit anti-MxA [47], rabbit antip-IRF3 (\#4947; 1:1000 dilution: Cell Signaling Technology, Inc., Beverly, MA, USA) and rabbit anti-p-Akt (\#9271 s; 1:1000 dilution: Cell Signaling Technology,) immunoglobulins were used. For anti-M1 and anti-NEP antibodies, Glutathione S-transferase (GST) -tagged A/Udorn/72 GST-M1 and polyhistidine-tagged A/Udorn/72 HisNEP (aa 11-121) were expressed in Escherichia coli, purified with Glutathione-Sepharose (Amersham Biosciences, Buckinghamshire, United Kingdom) or preparative SDS-PAGE, respectively, and used to immunize rabbits four times at 3-week intervals (100 $\mu \mathrm{g}$ or $60 \mu \mathrm{g}$ of antigen/ immunization, respectively). As secondary antibodies in Western blot HRP-conjugated rabbit anti-guinea pig (1:1000 dilution; Dako, Glostrup, Denmark) and goat anti-rabbit (1:2000 dilution; Dako) immunoglobulins were used.

\section{Immunofluorescence analysis}

A549 cells grown on glass coverslips were infected with the wt or NES-mutant viruses at a multiplicity of infection (MOI) -value of 1 and incubated at $37^{\circ} \mathrm{C} 5 \% \mathrm{CO}_{2}$ for $1 \mathrm{~h}$, after which the virus inoculum was removed and replaced with MEM containing 2\% FCS. Incubation was resumed and at $8 \mathrm{~h}, 12 \mathrm{~h}, 16 \mathrm{~h}, 20 \mathrm{~h}$ and $24 \mathrm{~h}$ postinfection infected cells were fixed with $3 \%$ paraformaldehyde at room temperature for $20 \mathrm{~min}$, permeabilized with $0,1 \%$ Triton X-100 for $5 \mathrm{~min}$ and processed for immunofluorescence microscopy as described previously [48]. The localization of NS1 and NP in the cells were visualized with anti-NS1 and anti-NP antibodies (1:100 dilution) and photographed on a Leica TCS NT confocal microscope. For the leptomycin B (LMB) experiment $2 \mathrm{ng} / \mathrm{ml}$ of LMB was added on A549 cells infected with wt virus at $3 \mathrm{~h}$ post-infection and the cells were fixed at $20 \mathrm{~h}$ post-infection and processed as described above.

\section{Viral growth kinetics}

Confluent monolayers of MDCK cells were inoculated with the wt or NES-mutant viruses at MOI values of 0.001 and incubated at $37^{\circ} \mathrm{C} 5 \% \mathrm{CO}_{2}$. At $1 \mathrm{~h}$ post-infection the virus suspensions were removed and replaced with serum-free MEM containing 2,5 $\mu \mathrm{g} / \mathrm{ml}$ TPCK-trypsin. Incubation was resumed and cell culture supernatant samples were collected at $12 \mathrm{~h}$ intervals until $48 \mathrm{~h}$ post-infection. Virus titers of the samples were measured by endpoint dilution assay on MDCK cells as per standard protocol. Briefly, serial tenfold dilutions till $10^{-8}$ of each sample was made and used to infect confluent MDCK-cells on 96-well plates maintained in serum-free MEM with 2,5 $\mu \mathrm{g} / \mathrm{ml}$ TPCK-trypsin.
Eight parallel wells for each dilution were used. After three days of incubation at $37^{\circ} \mathrm{C} 5 \% \mathrm{CO}_{2}$ the wells were inspected for cytopathic effect by light microscope and $\mathrm{TCID}_{50} / \mathrm{ml}$-values were calculated using the SpearmanKarber method.

\section{Infection experiments}

For analyzing interferon (IFN) gene expression A549 cells on 6-well plates were infected at MOI values of 10, 2 and 1 for $1 \mathrm{~h}$ followed by removal of the virus inoculum and addition of fresh medium $(2 \mathrm{ml} /$ well $)$. At $20 \mathrm{~h}$ postinfection cell culture supernatants were collected and IFN- $\beta$ and IFN- $\lambda 1$ levels were determined by IFN-specific EIA assays using commercial kits (PBL Interferon source) according to the instructions of the manufacturer. Infected cells were collected in $1 \mathrm{mM}$ EDTA, $10 \mathrm{mM}$ Tris- $\mathrm{HCl}$ and lysed by pulling through a 25G needle ten times. Protein concentrations of the MOI 1 lysates were analysed by the Bradford method and equal amounts of proteins at different dilutions $(10,5,1,0,5$ or $0,25 \mu \mathrm{g}$ of total protein/ lane) were loaded and separated on $12 \%$ SDS-PAGE. Proteins were transferred onto Immobilon $\mathrm{P}$ polyvinylidene fluoride membranes, stained $\left(1 \mathrm{~h}\right.$ at $\mathrm{RT}$ or overnight $+4^{\circ} \mathrm{C}$ for p-IRF3 and p-Akt) with antibodies against NS1, Actin, MxA, p-IRF3 and p-Akt followed by staining with HRPconjugated secondary antibodies for $1 \mathrm{~h}$ at RT. Protein bands were visualized on HyperMax films using an ECL plus system (GE Healthcare). Statistical significance of the IFN-production results were calculated by comparing samples to the corresponding wild-type virus samples using the students t-test. For analysis of viral protein expression kinetics, A549 cells were infected at MOI 1 as described above and cell lysates were collected at $2 \mathrm{~h}, 4 \mathrm{~h}$, $6 \mathrm{~h}, 8 \mathrm{~h}, 12 \mathrm{~h}$ and $24 \mathrm{~h}$ post-infection and immunoblotted against NS1, NP, M1, NEP and Actin as described above.

\section{NS1 binding assay}

The full length wild type A/Udorn/72 NS1 gene (V01102) was expressed in E. coli GST (pGEX-3X; Amersham Biosciences, Buckinghamshire, U. K.) expression vector. To create point mutations to NS1 cDNA, QuikChange ${ }^{\text {twi }}$ SiteDirected Mutagenesis Kit (Stratagene, La Jolla, CA, USA) was used. All DNA manipulations were performed according to standard protocols or as specified by the manufacturer, and the newly created gene constructs were sequenced. Influenza A virus GST-NS1 fusion proteins were expressed in E. coli BL21 cells, and GST-fusion proteins were purified in Glutathione-Sepharose as described [15]. In vitro-translated CPSF30 protein (TNT Coupled Reticulocyte Lysate Systems, Promega, Madison, WI, USA) were $\left[{ }^{35} \mathrm{~S}\right]$-labeled (PRO-MIX, Amersham Biosciences) and allowed to bind to Sepharose-immobilized GST or GSTNS1 fusion proteins on ice for $1 \mathrm{~h}$, followed by washing. GST-NS1-bound and $\left[{ }^{35} \mathrm{~S}\right]$-labeled proteins were 
separated on $12 \%$ SDS-PAGE. The gels were fixed and treated with Amplify reagent (Amersham Biosciences, Buckinghamshire, U. K.) as specified by the manufacturer and autoradiographed.

\section{Additional files}

Additional file 1: Figure S1. Intracellular localization of NP and NS1 on A549 cells. A549 cells were infected at MOI 1 for the times indicated with wild type, (L141A) and (L146A) recombinant viruses before fixation and permeabilization. Cells were stained with guinea pig anti-NS1 and rabbit anti-NP antibodies followed by Rhodamine Red X-labeled anti-guinea pig immunoglobulins and FITC-labeled anti-rabbit immunoglobulins. Pictures were taken with Leica TCS NT confocal microscope.

Additional file 2: Figure S2. Intracellular localization of NP and NS1 on A549 cells. A549 cells were infected at MOI 1 for the times indicated with (A149V), (L144A, L146A) and (F138A, L141A) recombinant viruses before fixation and permeabilization. Cells were stained with guinea pig anti-NS1 and rabbit anti-NP antibodies followed by Rhodamine Red X-labeled anti-guinea pig immunoglobulins and FITC-labeled anti-rabbit immunoglobulins. Pictures were taken with Leica TCS NT confocal microscope.

Additional file 3: Figure S3. Comparison of NS1 expression levels. A, lysates collected from infected A549 cells (MOI 1) at $20 \mathrm{~h}$ post-infection were analyzed by SDS-PAGE and Western blotting and the amounts of NS1 and actin were visualized by immunoblotting. Shown are three dilutions for each sample $(10,1,0,5$ or $0,25 \mu \mathrm{g}$ total protein/lane). B, bands from the $1 \mu \mathrm{g}$ lanes were processed with ImageJ software (http//imagej.nih.gov/ij/) to turn pixel intensity into optical density (OD). NS1 OD values were normalized to actin OD values and compared to wild type virus to get a relative estimate of NS1 expression levels between the viruses. Data shown is representative of two independent experiments.

\section{Competing interests}

The authors declare that they have no competing interests.

\section{Authors' contributions}

JT did the experiments, participated in the design and wrote the manuscript. KM gave hands-on guidance on the experiments and participated in the design of the experiments and writing of the manuscript. IJ participated in the design of the experiments and writing of the manuscript. All authors read and approved the final manuscript.

\section{Acknowledgements}

The authors wish to thank Dr. Robert A. Lamb for providing the plasmids necessary for generating A/Udorn/72 recombinant viruses and Dr. Robert Krug for the CPSF30 expression plasmid. This work was funded by the Medical Research Council of the Academy of Finland (grants 252252 and 255780) and the Sigrid Juselius Foundation.

\section{Author details}

'Virology Unit, Department of Infectious Disease Surveillance and Control, National Institute for Health and Welfare (THL), Mannerheimintie 166, FIN-00300 Helsinki, Finland. ${ }^{2}$ Department of Virology, University of Turku, Turku, Finland.

Received: 25 October 2013 Accepted: 4 July 2014

Published: 14 July 2014

\section{References}

1. Fukuyama S, Kawaoka Y: The pathogenesis of influenza virus infections: the contributions of virus and host factors. Curr Opin Immunol 2011, 23(4):481-486.

2. Hale BG, Randall RE, Ortin J, Jackson D: The multifunctional NS1 protein of influenza A viruses. J Gen Virol 2008, 89(Pt 10):2359-2376.

3. Gack MU, Albrecht RA, Urano T, Inn K, Huang I, Carnero E, Farzan M, Inoue S, Jung JU, Garcia-Sastre A: Influenza A virus NS1 targets the ubiquitin ligase TRIM25 to evade recognition by the host viral RNA sensor RIG-I. Cell Host Microbe 2009, 5(5):439-449.

4. Nemeroff M, Barabino S, Li Y, Keller W, Krug R: Influenza virus NS1 protein interacts with the cellular $30 \mathrm{kDa}$ subunit of CPSF and inhibits 3 ' end formation of cellular pre-mRNAs. Mol Cell 1998, 1(7):991-1000.

5. Chen Z, Li Y, Krug R: Influenza A virus NS1 protein targets poly(A)-binding protein II of the cellular 3 '-end processing machinery. EMBO J 1999, 18(8):2273-2283.

6. Li S, Min J, Krug R, Sen G: Binding of the influenza A virus NS1 protein to PKR mediates the inhibition of its activation by either PACT or double-stranded RNA. Virol 2006, 349(1):13-21.

7. Min J, Krug R: The primary function of RNA binding by the influenza A virus NS1 protein in infected cells: inhibiting the 2 ' -5 ' oligo (a) synthetase/RNase L pathway. Proc Natl Acad Sci U S A 2006, 103(18):7100-7105.

8. Li W, Li H, Lu R, Li F, Dus M, Atkinson P, Brydon E, Johnson K, Garcia-Sastre $A$, Ball $L$, Palese $P$, Ding S: Interferon antagonist proteins of influenza and vaccinia viruses are suppressors of RNA silencing. Proc Natl Acad Sci U S A 2004, 101(5):1350-1355.

9. Fortes P, Beloso A, Ortin J: Influenza-virus Ns1 protein inhibits premessenger Rna splicing and blocks messenger-Rna nucleocytoplasmic transport. EMBO J 1994, 13(3):704-712.

10. Garaigorta U, Ortin J: Mutation analysis of a recombinant NS replicon shows that influenza virus NS1 protein blocks the splicing and nucleocytoplasmic transport of its own viral mRNA. Nucleic Acids Res 2007, 35(14):4573-4582.

11. Aragon T, de la Luna S, Novoa I, Carrasco L, Ortin J, Nieto A: Eukaryotic translation initiation factor $4 \mathrm{Gl}$ is a cellular target for NS1 protein, a translational activator of influenza virus. Mol Cell Biol 2000, 20(17):6259-6268.

12. Hale BG, Jackson D, Chen Y, Lamb RA, Randall RE: Influenza A virus NS1 protein binds $\mathrm{p} 85$ beta and activates phosphatidylinositol-3-kinase signaling. Proc Natl Acad Sci U S A 2006, 103(38):14194-14199.

13. Greenspan D, Palese P, Krystal M: 2 nuclear location signals in the influenza-virus Ns1 nonstructural protein. J Virol 1988, 62(8):3020-3026.

14. Li Y, Yamakita Y, Krug R: Regulation of a nuclear export signal by an adjacent inhibitory sequence: the effector domain of the influenza virus NS1 protein. Proc Natl Acad Sci U S A 1998, 95(9):4864-4869.

15. Melen K, Kinnunen L, Fagerlund R, Ikonen N, Twu KY, Krug RM, Julkunen I: Nuclear and nucleolar targeting of influenza A virus NS1 protein: striking differences between different virus subtypes. J Virol 2007, 81(11):5995-6006.

16. Melen K, Tynell J, Fagerlund R, Roussel P, Hernandez-Verdun D, Julkunen I: Influenza A H3N2 subtype virus NS1 protein targets into the nucleus and binds primarily via its C-terminal NLS2/NoLS to nucleolin and fibrillarin. Virol J 2012, 9:167.

17. Sorokin AV, Kim ER, Ovchinnikov LP: Nucleocytoplasmic transport of proteins. Biochem-Moscow 2007, 72(13):1439-1457.

18. Holaska J, Black B, Love D, Hanover J, Leszyk J, Paschal B: Calreticulin is a receptor for nuclear export. J Cell Biol 2001, 152(1):127-140.

19. Holaska J, Black B, Rastinejad F, Paschal B: Ca2 +-dependent nuclear export mediated by calreticulin. Mol Cell Biol 2002, 22(17):6286-6297.

20. Han H, Cui Z, Wang W, Zhang Z, Wei H, Zhou Y, Zhang X: New regulatory mechanisms for the intracellular localization and trafficking of influenza A virus NS1 protein revealed by comparative analysis of $A / P R / 8 / 34$ and A/Sydney/5/97. J Gen Virol 2010, 91:2907-2917.

21. Squires RB, Noronha J, Hunt V, Garcia-Sastre A, Macken C, Baumgarth N, Suarez D, Pickett BE, Zhang Y, Larsen CN, Ramsey A, Zhou L, Zaremba S, Kumar S, Deitrich J, Klem E, Scheuermann RH: Influenza research database: an integrated bioinformatics resource for influenza research and surveillance. Influenza Other Respir Viruses 2012, 6(6):404-416.

22. Xia S, Monzingo AF, Robertus JD: Structure of NS1A effector domain from the influenza A/Udorn/72 virus. Acta Crystallogr D Biol Crystallogr 2009, 65(Pt 1):11-17

23. Li Z, Jiang Y, Jiao $P$, Wang A, Zhao F, Tian G, Wang X, Yu K, Bu Z, Chen H: The NSI gene contributes to the virulence of $\mathrm{H} 5 \mathrm{~N} 1$ avian influenza viruses. J Virol 2006, 80(22):11115-11123.

24. O'Neill RE, Talon J, Palese P: The influenza virus NEP (NS2 protein) mediates the nuclear export of viral ribonucleoproteins. EMBO J 1998, 17(1):288-296.

25. Twu K, Noah D, Rao P, Kuo R, Krug R: The CPSF30 binding site on the NS1A protein of influenza A virus is a potential antiviral target. J Virol 2006, 80(8):3957-3965. 
26. Nishi K, Yoshida M, Fujiwara D, Nishikawa M, Horinouchi S, Beppu T: Leptomycin-B targets a regulatory cascade of $\mathrm{Crm} 1$, a fission yeast nuclear-protein, involved in control of higher-order chromosome structure and gene-expression. J Biol Chem 1994, 269(9):6320-6324

27. Elton D, Simpson-Holley M, Archer K, Medcalf L, Hallam R, McCauley J, Digard P: Interaction of the influenza virus nucleoprotein with the cellular CRM1-mediated nuclear export pathway. J Virol 2001, 75(1):408-419.

28. Darapaneni $\mathrm{V}$, Prabhaker VK, Kukol A: Large-scale analysis of influenza A virus sequences reveals potential drug target sites of non-structural proteins. J Gen Virol 2009, 90:2124-2133.

29. Chua MA, Schmid S, Perez JT, Langlois RA, Tenoever BR: Influenza A virus utilizes suboptimal splicing to coordinate the timing of infection. Cell Rep 2013, 3(1):23-29.

30. Das K, Ma L, Xiao R, Radvansky B, Aramini J, Zhao L, Marklund J, Kuo R, Twu KY, Arnold E, Krug RM, Montelione GT: Structural basis for suppression of a host antiviral response by influenza A virus. Proc Natl Acad Sci U S A 2008, 105(35):13093-13098.

31. Min JY, Li S, Sen GC, Krug RM: A site on the influenza A virus NS1 protein mediates both inhibition of PKR activation and temporal regulation of viral RNA synthesis. Virology 2007, 363(1):236-243.

32. Martin $\mathrm{K}$, Helenius $A$ : Nuclear transport of influenza virus ribonucleoproteins: the viral matrix protein (M1) promotes export and inhibits import. Cell 1991, 67(1):117-130.

33. Ehrhardt C, Marjuki H, Wolff T, Nuernberg B, Planz O, Pleschka S, Ludwig S: Bivalent role of the phosphatidylinositol-3-kinase (PI3K) during influenza virus infection and host cell defence. Cell Microbiol 2006, 8(8):1336-1348.

34. Ehrhardt C, Wolff T, Pleschka S, Planz O, Beermann W, Bode JG, Schmolke M, Ludwig S: Influenza A virus NS1 protein activates the PI3K/Akt pathway to mediate antiapoptotic signaling responses. J Virol 2007, 81(7):3058-3067.

35. Shin Y, Li Y, Liu Q, Anderson DH, Babiuk LA, Zhou Y: SH3 binding motif 1 in influenza a virus NS1 protein is essential for PI3K/Akt signaling pathway activation. J Virol 2007, 81(23):12730-12739.

36. Zhirnov OP, Klenk $\mathrm{H}$ : Control of apoptosis in influenza virus-infected cells by up-regulation of Akt and p53 signaling. Apoptosis 2007, 12(8):1419-1432.

37. Shin Y, Liu Q, Tikoo SK, Babiuk LA, Zhou Y: Influenza A virus NS1 protein activates the phosphatidylinositol 3-kinase (PI3K)/Akt pathway by direct interaction with the p85 subunit of PI3K. J Gen Virol 2007, 88:13-18.

38. Hrincius ER, Dierkes $R$, Anhlan D, Wixler V, Ludwig S, Ehrhardt C: Phosphatidylinositol-3-kinase (PI3K) is activated by influenza virus vRNA via the pathogen pattern receptor Rig-I to promote efficient type I interferon production. Cell Microbiol 2011, 13(12):1907-1919.

39. Matsuda M, Suizu F, Hirata N, Miyazaki T, Obuse C, Noguchi M: Characterization of the interaction of influenza virus NS1 with Akt. Biochem Biophys Res Commun 2010, 395(3):312-317.

40. Li Y, Anderson DH, Liu Q, Zhou Y: Mechanism of influenza A virus NS1 protein interaction with the p85 beta, but not the p85 alpha, subunit of phosphatidylinositol 3-kinase (PI3K) and up-regulation of PI3K activity. J Biol Chem 2008, 283(34):23397-23409.

41. Hale BG, Kerry PS, Jackson D, Precious BL, Gray A, Killip MJ, Randall RE, Russell RJ: Structural insights into phosphoinositide 3-kinase activation by the influenza A virus NS1 protein. Proc Natl Acad Sci U S A 2010, 107(5):1954-1959.

42. Shin Y, Liu Q, Tikoo SK, Babiuk LA, Zhou Y: Effect of the phosphatidylinositol 3-kinase/Akt pathway on influenza A virus propagation. J Gen Virol 2007, 88:942-950.

43. Fan S, Macken CA, Li C, Ozawa M, Goto H, Iswahyudi NF, Nidom CA, Chen H, Neumann G, Kawaoka Y: Synergistic effect of the PDZ and p85beta-binding domains of the NS1 protein on virulence of an avian H5N1 influenza A virus. J Virol 2013, 87(9):4861-4871

44. Li W, Wang G, Zhang H, Shen Y, Dai J, Wu L, Zhou J, Jiang Z, Li K: Inability of NS1 protein from an H5N1 influenza virus to activate PI3K/Akt signaling pathway correlates to the enhanced virus replication upon PI3K inhibition. Vet Res 2012, 43:36-9716. 43-36.

45. Neumann G, Watanabe T, Ito H, Watanabe $S$, Goto H, Gao P, Hughes M, Perez D, Donis R, Hoffmann E, Hobom G, Kawaoka Y: Generation of influenza A viruses entirely from cloned cDNAs. Proc Natl Acad Sci U S A 1999, 96(16):9345-9350.

46. Ronni T, Sareneva T, Pirhonen J, Julkunen I: Activation of Ifn-alpha, Ifn-gamma, Mxa, and Ifn regulatory factor-1 genes in influenza-a virus-infected human peripheral-blood mononuclear-cells. J Immunol 1995, 154(6):2764-2774.
47. Ronni T, Melen K, Malygin A, Julkunen I: Control of Ifn-inducible Mxa gene-expression in human-cells. J Immunol 1993, 150(5):1715-1726.

48. Melen K, Keskinen P, Ronni T, Sareneva T, Lounatmaa K, Julkunen I: Human MxB protein, an interferon-alpha-inducible GTPase, contains a nuclear targeting signal and is localized in the heterochromatin region beneath the nuclear envelope. J Biol Chem 1996, 271(38):23478-23486.

doi:10.1186/1743-422X-11-128

Cite this article as: Tynell et al:: Mutations within the conserved NS1 nuclear export signal lead to inhibition of influenza A virus replication. Virology Journal 2014 11:128.

\section{Submit your next manuscript to BioMed Central and take full advantage of:}

- Convenient online submission

- Thorough peer review

- No space constraints or color figure charges

- Immediate publication on acceptance

- Inclusion in PubMed, CAS, Scopus and Google Scholar

- Research which is freely available for redistribution

Submit your manuscript at www.biomedcentral.com/submit
C Biomed Central 